\section{IOSH2 EXERTS POTENT ANTI-TUMOR ACTIVITY BY BLOCKING LILRB1/2 AND KIR3DL1 RECEPTOR SIGNALING}

${ }^{1}$ Osiris Marroquin Belaunzaran, ${ }^{1}$ Anahita Rafiei*, ${ }^{1}$ Anil Kumar, ${ }^{1}$ Marco Gualandi, ${ }^{1}$ Magdalena Westphal, 'Lorenz Vogt, 'Sean Smith, ${ }^{2}$ Michael Curran, ${ }^{1}$ Christoph Renner. ${ }^{1}$ ImmunOs Therapeutics AG, Schlieren, Switzerland; ${ }^{2}$ The UTD MD Anderson Cancer, Houston, TX, USA

Background To develop novel anti-cancer therapeutics we have used a reverse rational approach and searched for human HLA class I molecules known to induce autoimmunity and long-term lasting viral control as a surrogate marker for potential anti-cancer activity. HLA-B*27 or HLA-B*57 are well known genetic factors associated with superior control of viral infections (e.g. HIV and HCV) through processes related to both adaptive and innate immunity. Here we demonstrate that the expression of an optimised HLA-B57-Fc fusion protein (iosH2) exerts anti-tumor efficacy through its multimodal inhibition of LILRB1/2 and KIR3DL1 receptors.

Methods iosH2 was produced by stable expression in $\mathrm{CHO}$ cells and purified by standard chromatography techniques. Interaction and competition studies were performed using BioLayer Interferometry, ELISA, and cell-based assays. Analysis of LILRB1/2 downstream ITIM signaling was assessed using an automated western blot system. Functional cell-based assays including in vitro polarization and phagocytosis of macrophages, T cell and NK cell assays were assessed using live-cell imaging. In vivo efficacy studies were performed using syngeneic and humanized mouse models of cancer.

Results iosH2 binds with nanomolar affinity to LILRB1/2 and KIR3DL1, and blocks HLA-G and ANGPTL's binding to LILRB1/2. iosH2 reduces ITIM downstream signalling including phosphorylation of SHP1/2 and promotes conversion from M2 to M1 macrophage phenotype resulting in enhanced tumor cell phagocytosis in vitro. In addition, ios $\mathrm{H} 2$ increases $\mathrm{T}$ and $\mathrm{NK}$ cell cytotoxicity in co-cultures with cancer cell lines. In vivo efficacy studies demonstrate therapeutic efficacy in syngeneic C38 colon cancer mice and in BRGSF-HIS humanized PDX NSCLC mice in concert with reduction of pro-tumorigenic cytokines.

Conclusions iosH2 binds to LILRB1/2 and KIR3DL1, restores immune effector cell function in vitro and demonstrates anti-tumor activity in diverse in vivo mouse models. iosH2 is a first-in-class multi-functional agent that promotes key components of the innate and adaptive immune system leading to profound anti-tumor activity. Clinical development is underway and a phase I trial in preparation.

Ethics Approval 1. Animal housing and experimental procedures were conducted according to the French and European Regulations and the National Research Council Guide for the Care and Use of Laboratory Animals7-8. The animal facility is authorized by the French authorities (Agreement $\mathrm{N}^{\circ}$ B 21 231011 EA). All animals procedures (including surgery, anesthesia and euthanasia as applicable) used in the current study (200269/ACT1 C38 SC/Ethical protocol: ONCO 1) were submitted to the Institutional Animal Care and Use Committee of Oncodesign (Oncomet) approved by French authorities (CNREEA agreement $\mathrm{N}^{\circ}$ 91). 2. Animal welfare for this study complies with the UK Animals Scientific Procedures Act 1986 (ASPA) in line with Directive 2010/63/EU of the European Parliament and the Council of 22 September 2010 on the protection of animals used for scientific purposes. All experimental data management and reporting procedures were in strict accordance with applicable Crown Bioscience UK Guidelines and Standard Operating Procedures.

http://dx.doi.org/10.1136/jitc-2021-SITC2021.865 